\title{
Risk Factors for Mortality in Melioidosis: A Single-Centre, 10-Year Retrospective Cohort Study
}

\author{
Raviraj Menon $\left(\mathbb{D},{ }^{1}\right.$ Poornima Baby $\mathbb{D D}^{2}$, Anil Kumar V. (iD, ${ }^{2}$ Sandeep Surendran $\left(\mathbb{D},{ }^{3}\right.$ \\ Manu Pradeep $\mathbb{D},{ }^{4}$ Aiswarya Rajendran $\mathbb{D},{ }^{5}$ Gaayathri Suju $\mathbb{D},{ }^{6}$ and Arathy Ashok $\mathbb{D}^{2}$ \\ ${ }^{1}$ Department of General Medicine, Amrita Institute of Medical Sciences, Amrita Vishwa Vidyapeetham, Kochi, Kerala, India \\ ${ }^{2}$ Department of Microbiology, Amrita Institute of Medical Sciences, Amrita Vishwa Vidyapeetham, Kochi, Kerala, India \\ ${ }^{3}$ Department of Rheumatology, Amrita Institute of Medical Sciences, Amrita Vishwa Vidyapeetham, Kochi, Kerala, India \\ ${ }^{4}$ Department of Community Medicine, Amrita Institute of Medical Sciences, Amrita Vishwa Vidyapeetham, Kochi, Kerala, India \\ ${ }^{5}$ University of Massachusetts, Amherst, MA, USA \\ ${ }^{6}$ Amrita School of Medicine, Amrita Institute of Medical Sciences, Amrita Vishwa Vidyapeetham, Kochi, Kerala, India
}

Correspondence should be addressed to Manu Pradeep; drmanupradeep24@gmail.com

Received 1 May 2021; Accepted 19 June 2021; Published 5 July 2021

Academic Editor: Ahmet Baydin

Copyright (C 2021 Raviraj Menon et al. This is an open access article distributed under the Creative Commons Attribution License, which permits unrestricted use, distribution, and reproduction in any medium, provided the original work is properly cited.

\begin{abstract}
Melioidosis is a tropical infectious disease with diverse clinical presentations. We aimed to investigate the characteristics and mortality risk factors of patients diagnosed with melioidosis in the past 10 years. This was a retrospective cohort study conducted at a quaternary care centre in South India. Clinical, demographic, and biochemical data in patients diagnosed with melioidosis with cultures were collected between January 2011 and December 2020 from medical records. Logistic regression analysis was performed to screen mortality risk factors of melioidosis in addition to descriptive statistics and chi-square analysis. Seventy-three melioidosis patients' records were analysed, and the most common comorbidity was type 2 diabetes mellitus $(n=53,72.6 \%)$. The patients showed diverse presentations: pulmonary involvement, 30 (41.1\%); splenomegaly, 29 (39.7\%); abscesses and cutaneous involvement, 18 (24.7\%); lymph node, 10 (13.7\%); arthritis and osteomyelitis, 9 (12.3\%); and genitourinary infection, 4 (5.4\%). The mortality was noted to be 15 (20.5\%). Logistic regression analysis indicated that chronic kidney disease $(\mathrm{OR}=14.0), \mathrm{CRP}>100 \mathrm{IU} /$ $\mathrm{L}(\mathrm{OR}=6.964)$, and $\mathrm{S}$. albumin $<3 \mathrm{gm} / \mathrm{dl}(\mathrm{OR}=8.0)$ were risk factors associated with mortality and can guide in risk stratification. Hypoalbuminemia is a novel mortality risk factor, detected in this study, and requires further investigation to validate its utility as a prognostic marker and reveal possible therapeutic benefits in clinical correction.
\end{abstract}

\section{Introduction}

Melioidosis is an infection caused by the Gram-negative bacterium Burkholderia pseudomallei, which is a free-living organism found in surface water of rice paddies, freshly cultivated fields oil palm, drains, gardens, and playgrounds in endemic areas [1]. It is classified as a Class B Bioterrorism agent by the CDC and given the second highest priority [2]. Mode of transmission is commonly through direct human contact with infected soil or water, either by inhalation and cutaneous inoculation or by ingestion [3]. The leading risk factor for the disease is type 2 diabetes mellitus (DM), while other common risk factors include malignancy, chronic kidney disease, and immunosuppressive treatment $[4,5]$. Clinical features differ considerably, from acute pulmonary or septicemic presentations that are often fatal, to chronic localized infection that can then worsen to acute sepsis, with B. pseudomallei cultured from blood, pus, urine, and other bodily tissues and secretions. Presentation can also vary, notably as a high fever, with abscesses in the lungs, spleen, and liver as well as bone and joint involvement [1]. According to a systematic review conducted in 2015, looking at clinical presentations of 8469 patients, pneumonia (35.7\%), intra-abdominal abscess (18.3\%), and sepsis (18\%) were determined to be leading outcomes [6]. These confusing presentations of melioidosis demand diagnosis by 
microbiological confirmation [7]. High-sensitivity serological tests such as indirect hemagglutination assay (IHA), enzyme-linked immunosorbent assay (ELISA), and dot immunoassay have the potential to be used for screening for the infection in endemic communities but tissue culture remains the gold standard for diagnostic confirmation [8].

Melioidosis is a disease of alarming fatality, with overall mortality rates varying between 19 and 54\% in different endemic regions [9]. The recommended antimicrobial therapy includes ceftazidime, trimethoprim/sulfamethoxazole, and doxycycline [1], but established melioidosis is hard to treat, leading to unprecedented patient demises [10]. A review of the published literature from cohorts in Australia and Thailand showed that age above 50 years, chronic kidney disease, and development of septicemia were predictors of mortality. A majority of melioidosis cases have been reported from North Eastern Thailand and North Australia, but it has been emerging steadily in the Indian subcontinent, China, the Middle East, Africa, and South America [11]. The west coast of India is particularly vulnerable as the climate, population density, and presence of risk factors among the populace favour endemicity [12]. Unfortunately, further data regarding epidemiology and risk factors for mortality in Indian patients are scanty since diagnosis and reporting are uncoordinated and sporadic. Hence, further investigation is warranted in Indian population.

The primary objective of this cohort was to study the clinical characteristics and biochemical markers and investigate for risk factors that may be associated with mortality in patients diagnosed with melioidosis in a quaternary care referral centre over the past 10 years.

\section{Materials and Methods}

\subsection{Study Design and Inclusion and Exclusion Criteria.} Designed as a retrospective cohort study, all patients who were diagnosed as having melioidosis at a quaternary care centre in the west coast of South India between January 1, 2011, and December 31, 2020, were considered. Patients were included in the study, if they were more than 18 years of age and had grown B. pseudomallei from culture samples done in the in-house microbiological laboratory. Patients were excluded, if their blood or tissue cultures were done by external laboratories or if there were insufficient clinical or demographic details in their medical records.

\subsection{Identification of Isolates and Antimicrobial Susceptibility} Testing. B. pseudomallei was grown from samples like blood, pus aspirate, tissue, sputum, pleural fluid, throat swab, synovial fluid, or urine as dry wrinkled oxidase positive colonies on 5\% sheep blood agar and MacConkey agar media. On Gram staining, the organism appeared as a Gramnegative bacillus with bipolar staining. B. pseudomallei reduced nitrate, dihydrolysed arginine, and utilised glucose and lactose oxidatively. The isolates were further identified and the antimicrobial susceptibility testing was performed using the VITEK ${ }^{\circledR 2}$ Compact system (bioMérieux).
2.3. Data Collection. All data were retrospectively compiled from the Electronic Medical Record (EMR) of the patients who satisfied the inclusion and exclusion criteria. Demographic data (age and gender) and comorbidities (DM, coronary artery disease, and hypertension, chronic kidney disease, malignancy) of all included participants were compiled. Clinical presentations were classified into groups by two authors to account for reporting bias, and in case of a dispute a third author's decision was used. Based on the primary organ affected at presentation, patient data was classified into (1) pulmonary: including pneumonia (radiological changes on X-ray or CT), pleural effusion, and lung abscess; (2) cutaneous: including soft tissue infections, and infections of nonskeletal tissue surrounding or supporting organs and other structures including subcutaneous tissue and muscle; (3) genitourinary infection of the genital organs and the urinary tract including the kidneys; (4) visceral abscesses; (5) osteomyelitis; and (6) septic arthritis, as well as splenomegaly and lymph nodal involvement. Additionally, key biochemical parameters were extracted and analysed including white blood cells (WBC) count, hemoglobin, erythrocyte sedimentation rate (ESR), C-reactive protein (CRP), albumin, and alkaline phosphatase. Mortality for each patient was also collected from death summaries from the EMR system. This study will acknowledge selection bias, as patient data is obtained from a quaternary care referral centre, but no correction is applied to address it.

2.4. Statistical Methods. Descriptive statistics included frequency analysis (percentages) for categorical variables and means \pm standard deviations (SD) or median for continuous variables. Comparisons were determined by Student's $t$-test for continuous variables as appropriate and by the use of the $\chi 2$ test or Fisher exact test for categorical variables. Univariate logistic regression was performed to explore the association of significant parameters and estimate the mortality risk. The backward conditional method was used to select imaging variables entering the scoring system. The statistical significance level was set at 0.05 (two-tailed). All analyses were conducted with SPSS version 23.0 statistical software.

\section{Results}

This report describes 73 patients who were diagnosed with melioidosis at a quaternary care centre in South India between January 1st, 2011, and December 31st, 2020, after applying the inclusion and the exclusion criteria. Out of these, $65(89 \%)$ needed inpatient care and $8(11 \%)$ were managed on an outpatient basis. The mean $( \pm S D)$ age was $49.55 \pm 15.39$ years. The median length of stay was 15 days (1-139 days). The basic clinical details along with the biochemical parameters are summarized in Table 1. Table 1 also shows a comparison of the present cohort with other South Indian cohorts of melioidosis [13, 14].

Seventy-one (97.2\%) isolates of B. pseudomallei were found to be sensitive to ceftazidime, trimethoprim- 
TABLE 1: Comparison between demographic, clinical, and laboratory findings of the present study against other single-centre South Indian cohorts.

\begin{tabular}{|c|c|c|c|}
\hline Parameter & Present cohort & Saravu et al. [14] & Malladi et al. [13] \\
\hline Number of patients & 73 & 25 & 17 \\
\hline Region & Kerala & Karnataka $>$ Kerala & Andhra Pradesh \\
\hline Age in years & $49.5 \pm 15.36$ & $47.9 \pm 18.5$ & - \\
\hline \multicolumn{4}{|l|}{ Gender } \\
\hline Male & $55(75.3 \%)$ & 15 & 13 \\
\hline Female & $18(24.7 \%)$ & 10 & 4 \\
\hline \multicolumn{4}{|l|}{ Comorbidities } \\
\hline $\mathrm{DM}$ & $53(72.6 \%)$ & $19(76 \%)$ & $16(94 \%)$ \\
\hline Hypertension & $24(32.8 \%)$ & NA & - \\
\hline Coronary artery disease & 10 & NA & - \\
\hline Chronic kidney disease & 7 & $3(12 \%)$ & - \\
\hline Malignancy & 5 & NA & - \\
\hline Duration of symptoms & 1 day to 4 months & 2 days to 1 year & - \\
\hline Bacteremia & 34 & 13 & 12 \\
\hline \multicolumn{4}{|l|}{ Organ involvement } \\
\hline Pulmonary & $30(41.1 \%)$ & $9(36 \%)$ & $13(76 \%)$ \\
\hline Cutaneous & $18(24.7 \%)$ & - & $10(59 \%)$ \\
\hline Genitourinary & $4(5.4 \%)$ & - & - \\
\hline Arthritis & $9(12.3 \%)$ & $3(12 \%)$ & - \\
\hline Osteomyelitis & $9(12.3 \%)$ & 0 & - \\
\hline Abscess & $18(24.7 \%)$ & $4(16 \%)$ & $7(41 \%)$ \\
\hline Lymph node & $10(13.7 \%)$ & $2(8 \%)$ & - \\
\hline Splenomegaly & $29(39.7 \%)$ & - & - \\
\hline \multicolumn{4}{|l|}{ Key biochemical parameters } \\
\hline WBC count $(\times 103$ cells/cu.mm $)$ & $13.52 \pm 7.28$ & - & - \\
\hline Hemoglobin $(\mathrm{gm} / \mathrm{dl})$ & $10.78 \pm 2.29$ & - & - \\
\hline $\operatorname{ESR}\left(\mathrm{mm}\right.$ in the $\left.1^{\text {st }} \mathrm{hr}\right)$ & $57.86 \pm 22.618$ & - & - \\
\hline CRP (IU/L) & $126.46 \pm 89.01$ & - & - \\
\hline S. albumin $(\mathrm{gm} / \mathrm{dl})$ & $4.47 \pm 0.76$ & - & - \\
\hline $\operatorname{ALP}(\mathrm{mg} / \mathrm{dl})$ & $159.51 \pm 137.54$ & - & - \\
\hline Mortality & $15(20.547 \%)$ & - & $6(35.294 \%)$ \\
\hline
\end{tabular}

sulfamethoxazole, chloramphenicol, and doxycycline. Sixtyone $(83.5 \%)$ isolates were sensitive to imipenem, while sixtytwo $(85 \%)$ isolates were sensitive to tigecycline. The cohort was then stratified for mortality and analysed (Table 2 ). The three factors that showed significance underwent univariate analysis (Table 3). Chronic kidney disease $(\mathrm{OR}=14)$, C-reactive protein $>100 \mathrm{IU} / \mathrm{L}$ (OR 6.964), and S. albumin $<3 \mathrm{gm} / \mathrm{dl}(\mathrm{OR}=8)$ were detected to be risk factors for mortality in patients.

\section{Discussion}

This retrospective cohort is based on data of patients diagnosed with melioidosis over the past 10 years in Amrita Institute of Medical Sciences, Kochi, a quaternary referral and training centre in South India. The clinical and epidemiological profile and mortality risk factors of patients diagnosed with melioidosis during this period were assessed and characterised. A total of 73 patients were diagnosed to have melioidosis, of which 65 patients were managed as inpatients in the hospital. Fifty-five patients (75.3\%) were males with an average age of $49.5 \pm 15.36$ years at presentation, which was comparable with studies done by Saravu et al., 2010, and Currie et al., 2004 (Table 1). The reason for male preponderance may be explained by more frequent exposure to soil and water while in professions like farming or greater incidence of alcoholism among males $[9,15]$. The most common comorbid condition was DM with 53 (72.6\%) patients, followed by hypertension in 24 (32.8\%) patients. The prevalence of DM detected in this study is comparable to those of other cohorts $(74-80 \%)[7,12,16]$. The average duration of symptoms on presentation ranged from 1 day to 4 months with a wide variety of clinical presentations noted within our cohort. Pulmonary involvement was the most common presentation with 30 cases (41.1\%) which is comparable to lung involvement in Birnie et al., 2019 (35.7\%), followed by splenomegaly, 29 (39.7\%), abscesses and cutaneous involvement, 18 (24.7\%), lymph node enlargement, 10 (13.7\%), arthritis and osteomyelitis, 9 (12.3\%) and genitourinary infection, 4 (5.4\%) (Table 2). In contrast to sepsis reported by Birnie et al., 2019 (18\%), this cohort reported a much higher $46.5 \%$ of patients presenting with sepsis. The median length of stay in hospital was 15 days (1-159 days).

The mortality in this cohort was noted to be $20.5 \%$. The clinical presentation and mortality rates were comparable to those of a study done in Australia where the overall mortality was $19 \%$ with higher mortality in patients having CKD (31\% 
TAвLE 2: Demographic, clinical, and laboratory results stratified for mortality.

\begin{tabular}{|c|c|c|c|c|}
\hline Parameter & Total $(n=73)$ & Not expired $(n=58)$ & Expired $(n=15)$ & $P$ value \\
\hline \multicolumn{5}{|l|}{ Sociodemographic variables } \\
\hline Age & $49.5 \pm 15.36$ & $50.95 \pm 13.97$ & $44.13 \pm 19.48$ & 0.219 \\
\hline \multicolumn{5}{|l|}{ Gender } \\
\hline Male & 55 & 43 & 12 & 0.639 \\
\hline Female & 18 & 15 & 3 & \\
\hline \multicolumn{5}{|l|}{ Comorbidities } \\
\hline DM & 53 & 44 & 9 & 0.220 \\
\hline Hypertension & 24 & 19 & 5 & 0.996 \\
\hline Coronary artery disease & 10 & 7 & 3 & 0.419 \\
\hline Chronic kidney disease & 7 & 2 & 5 & 0.003 \\
\hline Malignancy & 5 & 3 & 2 & 0.271 \\
\hline \multicolumn{5}{|l|}{ Clinical and biochemical characteristics } \\
\hline \multicolumn{5}{|c|}{ Duration of symptoms before diagnosis } \\
\hline More than 14 days & 42 & 34 & 8 & 0.712 \\
\hline Less than and equal to 14 days & 31 & 24 & 7 & \\
\hline \multicolumn{5}{|l|}{ Organ involvement } \\
\hline Pulmonary & 30 & 21 & 9 & 0.095 \\
\hline Cutaneous & 18 & 16 & 2 & 0.330 \\
\hline Genitourinary & 4 & 2 & 2 & 0.185 \\
\hline Arthritis & 9 & 8 & 1 & 0.675 \\
\hline Abscess & 18 & 16 & 2 & 0.330 \\
\hline Osteomyelitis & 9 & 9 & 0 & 0.189 \\
\hline Lymph node & 10 & 10 & 0 & 0.110 \\
\hline Splenomegaly & 29 & 25 & 4 & 0.549 \\
\hline \multicolumn{5}{|l|}{ Biochemical parameters } \\
\hline Neutrophilic leucocytosis & 27 & 24 & 3 & 0.147 \\
\hline $\mathrm{ESR}>70 \mathrm{~mm} / \mathrm{hr}$ & 17 & 11 & 6 & 0.086 \\
\hline $\mathrm{CRP}>100 \mathrm{IU} / \mathrm{L}$ & 41 & 28 & 13 & 0.008 \\
\hline Albumin $<3 \mathrm{gm} / \mathrm{dl}$ & 29 & 26 & 3 & 0.004 \\
\hline ALP $>110 \mathrm{mg} / \mathrm{dl}$ & 41 & 32 & 9 & 0.737 \\
\hline
\end{tabular}

TABLE 3: Univariate analysis of mortality risk factors in melioidosis.

\begin{tabular}{lcc}
\hline \multirow{2}{*}{ Parameter } & \multicolumn{2}{c}{ Univariate analysis } \\
& $P$ value & Odds ratio $(95 \% \mathrm{CI})$ \\
\hline Chronic kidney disease & 0.004 & $14.000(2.379-82.398)$ \\
CRP $>100 \mathrm{IU} / \mathrm{L}$ & 0.016 & $6.964(1.441-33.652)$ \\
S. albumin $<3$ gm/dl & 0.010 & $8.000(1.654-38.688)$ \\
\hline
\end{tabular}

of total deaths) [17]. In our study, logistic regression analysis indicated that chronic kidney disease, C-reactive protein $>100 \mathrm{IU} / \mathrm{L}$, and serum albumin $<3 \mathrm{gm} / \mathrm{dl}$ were independent risk factors associated with mortality in melioidosis patients $-(\mathrm{OR}=14.0, \mathrm{OR}=6.964$, and $\mathrm{OR}=8.0$, respectively) (Table 3). This is in contrast to data from previous independent studies in Australia and Thailand where age more than 40 years, chronic kidney disease, occurrence of pneumonia, and septicemia were considered as predictors of mortality $[18,19]$.

Patients with chronic kidney disease have altered immune status with defective granulocyte, T-cells, and dendritic cell function, which predispose them to severe infection and increased mortality [20]. Low serum albumin levels result in increased mortality in patients with severe sepsis. This can be attributed to albumin being a carrier for several endogenous and exogenous molecules, with antioxidant and anti-inflammatory properties, and also it is the main protein involved in maintenance of plasma colloid osmotic pressure, all of which help to maintain hemodynamic stability. Albumin can also combine with free fatty acids in blood, which protects them from lipid peroxidation damage and it can also reduce or eliminate the toxicity of many exogenous toxic molecules by binding with them, which in turn reduces endothelial damage, as well as the risk of complications including mortality [21-23]. In contrast to the results of the present study, a 175-patient prospective study detected that CRP levels at admission were not found to be sensitive enough to categorise acute, chronic, or relapsed melioidosis in a suspected patient presenting with fever [24].

\section{Conclusion}

A limitation to note is that, as a quaternary care centre, with a greater number of referral cases from different hospitals, severity of the clinical outcome may show an institutional pattern which might not be applicable to population at large, thus incurring a selection bias. This may have led to an overestimation in the magnitude of impact of risk factors detected in the study, because the patient cases evaluated were of higher severity. Another limitation to note is the small sample size, but, relatively speaking, in context to the present Indian scenario, this study was analysed on a large 
patient data set with exhaustive biomarker records. The results discussed in this study may have external validity limited only to inpatient care in tertiary care centres, which remain the principal points of care in severe disease, equipped to diagnose and adequately manage melioidosis in India.

However, one should have a high index of suspicion for melioidosis and send appropriate cultures for the early diagnosis. Various risk factors for the disease should be identified at the earliest, and treatment should be initiated promptly after sending appropriate cultures. Patients having either chronic kidney disease or high CRP levels (more than $100 \mathrm{IU} / \mathrm{l}$ ) or hypoalbuminemia could be identified as highrisk patients and treatment must be continued under close monitoring to pick up complications that may lead to mortality. Based on the results of this cohort, S. albumin may emerge as a novel independent modifiable risk factor with further evidence and may serve as a crucial prognostic parameter for risk stratification in melioidosis patients. Further clinical trials with S. albumin correction (target serum conc. of $3 \mathrm{gm} / \mathrm{dl}$ ) as intervention can be safely tried in melioidosis patients presenting with sepsis. Patel et al., 2014, in a systematic review and meta-analysis, did not show a robust therapeutic benefit for human albumin in all-cause mortality of sepsis [25]. However, it is recommended that further large-scale trials be done to identify patient populations with microbiological evidence and build greater evidence with adequate analysis of biochemical markers associated with the high fatality rates of melioidosis associated sepsis.

\section{Data Availability}

The patient medical records' data used to support the findings of this study have been deposited in the Mendeley Data repository (DOI: 10.17632/x7tvmxnb5t.1).

\section{Ethical Approval}

The present study, including access to the patients' biological and registry data from the hospital information system, was approved by the Institutional Ethics Committee of the Amrita Institute of Medical Sciences, Kochi, India.

\section{Disclosure}

This original research was performed by the authors as part of employment at Amrita Institute of Medical Sciences, India.

\section{Conflicts of Interest}

The authors declare that there are no conflicts of interest regarding the publication of this paper.

\section{Acknowledgments}

The authors would like to extend their note of gratitude to the clinical and administrative staff of Amrita Institute of
Medical Sciences, Kochi, India, for facilitating the data collection process.

\section{References}

[1] M. Samuel and T. Ti, Interventions for Treating Melioidosis, Wiley, Hoboken, NJ, USA, 2002, https://www. cochranelibrary.com/cdsr/doi/10.1002/14651858.CD001263/ full.

[2] CDC Emergency Preparedness and Response. Bioterrorism Agents/Diseases. Centers for Disease Control and Prevention. 2018 https://emergency.cdc.gov/agent/agentlist-category.asp.

[3] A. Chrispal, S. J. Rajan, and S. Sathyendra, "The clinical profile and predictors of mortality in patients with melioidosis in South India," Tropical Doctor, vol. 40, no. 1, pp. 36-38, 2010.

[4] K. P. W. Chan, J. G. H. Low, J. Raghuram, S. M. C. FookChong, and A. Kurup, "Clinical characteristics and outcome of severe melioidosis requiring intensive care," Chest, vol. 128, no. 5, pp. 3674-3678, 2005.

[5] A. C. Cheng and B. J. Currie, "Melioidosis: epidemiology, pathophysiology, and management," Clinical Microbiology Reviews, vol. 18, pp. 383-416, 2005.

[6] E. Birnie, H. S. Virk, J. Savelkoel et al., "Global burden of melioidosis in 2015: a systematic review and data synthesis," The Lancet Infectious Diseases, vol. 19, no. 8, pp. 892-902, 2019.

[7] A. R. Zueter, C. Y. Yean, M. Abumarzouq, Z. A. Rahman, Z. Z. Deris, and A. Harun, "The epidemiology and clinical spectrum of melioidosis in a teaching hospital in a NorthEastern state of Malaysia: a fifteen-year review," BMC Infectious Diseases, vol. 16, no. 1, 2016.

[8] L. Fairley, S. Smith, S. Maisrikrod, and L. Henning, "Systematic review and meta-analysis of diagnostic tests for diagnosis of melioidosis," Acta Tropica, vol. 214, Article ID 105784, 2021.

[9] M. R. Abu Hassan, N. Aziz, N. Ismail et al., "Socio-epidemiological and land cover risk factors for melioidosis in Kedah, Northern Malaysia," PLOS Neglected Tropical Diseases, vol. 13, no. 3, Article ID e0007243, 2019.

[10] M. R. A. Hassan, S. P. Pani, N. P. Peng et al., "Incidence, risk factors and clinical epidemiology of melioidosis: a complex socio-ecological emerging infectious disease in the Alor Setar region of Kedah, Malaysia," BMC Infectious Diseases, vol. 10, 2010.

[11] S. J. Peacock, D. Limmathurotsakul, Y. Lubell et al., "Correction: melioidosis vaccines: a systematic review and appraisal of the potential to exploit biodefense vaccines for public health purposes," PLoS Neglected Tropical Diseases, vol. 7, no. 2, 2013.

[12] K. Vidyalakshmi, S. Lipika, S. Vishal, S. Damodar, and M. Chakrapani, "Emerging clinico-epidemiological trends in melioidosis: analysis of 95 cases from western coastal India," International Journal of Infectious Diseases, vol. 16, no. 7, 2012.

[13] S. V. S. Malladi, L. Vemu, N. C. Chandra et al., "Clinical spectrum of melioidosis at a tertiary care hospital in South India," International Journal of Infectious Diseases, vol. 45, no. April, p. 470, 2016.

[14] K. Saravu, C. Mukhopadhyay, S. Vishwanath et al., "Melioidosis in southern India: epidemiological and clinical profile," Southeast Asian Journal of Tropical Medicine and Public Health, vol. 41, no. 2, pp. 401-409, 2010.

[15] R. Gopalakrishnan, D. Sureshkumar, M. A. Thirunarayan, and V. Ramasubramanian, "Melioidosis: an emerging infection in 
India," Journal of the Association of Physicians of India, vol. 61, no. 9, pp. 612-614, 2013.

[16] E. George and A. Rajalakshmi, "Melioidosis-a case series from Kerala," Journal of the Association of Physicians of India, vol. 67, p. 40, 2019.

[17] B. J. Currie, D. A. Fisher, D. M. Howard et al., "Endemic melioidosis in tropical northern Australia: a 10-year prospective study and review of the literature," Clinical Infectious Diseases, vol. 31, no. 4, pp. 981-986, 2000.

[18] B. J. Currie, L. Ward, and A. C. Cheng, "The epidemiology and clinical spectrum of melioidosis: 540 cases from the 20 year Darwin prospective study," PLoS Neglected Tropical Diseases, vol. 4 , no. 11, 2010.

[19] V. Hantrakun, S. Kongyu, P. Klaytong et al., "Clinical epidemiology of 7126 melioidosis patients in Thailand and the implications for a national notifiable diseases surveillance system," Open Forum Infectious Diseases, vol. 6, no. 12, 2019.

[20] L. S. Dalrymple and A. S. Go, "Epidemiology of acute infections among patients with chronic kidney disease," Clinical Journal of the American Society of Nephrology, vol. 3, pp. 1487-1493, 2008.

[21] P. Caironi, G. Tognoni, S. Masson et al., "Albumin replacement in patients with severe sepsis or septic shock," The New England Journal of Medicine, vol. 370, no. 15, pp. 1412-1421, 2014.

[22] S.-Z. Qian, D. Jin, Z.-B. Chen et al., "Hypoalbuminemia, a novel prognostic factor for prediction of long-term outcomes in critically ill patients with septic shock," International Journal of Clinical and Experimental Medicine, vol. 12, no. 6, pp. 7401-7409, 2019.

[23] P. B. Soeters, R. R. Wolfe, and A. Shenkin, "Hypoalbuminemia: pathogenesis and clinical significance," Journal of Parenteral and Enteral Nutrition, vol. 43, pp. 181-193, 2019.

[24] A. C. Cheng, M. O’Brien, S. P. Jacups, N. M. Anstey, and B. J. Currie, "C-reactive protein in the diagnosis of melioidosis," The American Journal of Tropical Medicine and Hygiene, vol. 70, no. 5, pp. 580-582, 2004.

[25] A. Patel, M. A. Laffan, U. Waheed, and S. J. Brett, "Randomised trials of human albumin for adults with sepsis: systematic review and meta-analysis with trial sequential analysis of all-cause mortality," BMJ, vol. 349, 2014. 